\title{
DECISIONS
}

\section{A 52-year-old woman with a positive tuberculin skin test}

\author{
Vanessa J. Redditt MD, Paul E. Bunce MD
}

Either a tuberculin skin test or an interferon- $\gamma$ release assay are appropriate tests to screen for latent TB infection in this patient. ${ }^{2}$ The newer interferon- $\gamma$ release assays offer benefits compared with standard skin testing (i.e., single-visit testing, improved specificity, not affected by BCG vaccination), but are more expensive and are often less accessible to family physicians. ${ }^{2,4}$ In situations with increased risks of infection, disease progression or poor clinical outcomes (as in this case), both tests may be used to enhance overall sensitivity: if the result of the initial tuberculin skin test is negative, then an interferon- $\gamma$ release assay could be performed (or vice versa). ${ }^{2}$ These tests are not recommended for the diagnosis of active TB in adults. \section{for latent TB infection? If so, was the appropriate test chosen?}

Should this patient have been tested

This patient emigrated from a country with elevated rates of TB and is immunocompromised. She is at increased risk of development of active TB and, therefore, was appropriately tested for latent TB infection.

Following infection with TB, there is an estimated $10 \%$ lifetime risk of progression to active disease, with half occurring during the first two years after infection. ${ }^{1}$ Three main categories of people should be tested for latent TB infection: those with recent $\mathrm{TB}$ infection or exposure; those with impaired immunity; and those with radiographic evidence of prior TB but no previous treatment. ${ }^{2}$ Testing is appropriate only if there is a commitment to treat the patient if results are positive.

Appendix 1 (available at www.cmaj.ca/lookup /suppl/doi:10.1503/cmaj.131042/-/DC1) summarizes risk factors associated with progression to active TB among people with a positive tuberculin skin test. Compared with people with no known risk factor, people at high risk for progression include those with HIV/AIDS, transplants, chronic renal failure requiring hemodialysis, a recent TB infection or chest radiograph showing fibronodular disease. People with diabetes mellitus, who are taking glucocorticoids $(\geq 15 \mathrm{mg} / \mathrm{d}$ ) or who are between 0 and 4 years of age are at moderate risk, and those with heavy alcohol consumption, who smoke ( $\geq 1 \mathrm{pack} / \mathrm{d})$, are underweight, or have a chest radiograph showing a granuloma are at slightly increased risk.

\section{How should the result of the tuberculin skin test be interpreted?}

Based on the patient's risk category and current Canadian TB guidelines, ${ }^{2}$ the patient's tuberculin skin test is considered positive. The tuberculin skin test is interpreted according to the size of induration, positive predictive value and risk of progression to active TB. Induration measuring $10 \mathrm{~mm}$ or more, which has a sensitivity of $90 \%$ and specificity of $95 \%$, is a suitable criterion for most clinical situations in Canada. ${ }^{2}$ A criterion of 5-9 mm induration (with sensitivity $>98 \%$, but lower specificity) is recommended for patients with HIV infection, immunosuppression, end-stage renal disease, contact with active TB within two years and abnormal chest radiograph, as well as for children. ${ }^{2}$ A lower threshold may be considered for patients with both HIV immunosuppression and a high likelihood of TB infection. An online tool can results (http://tstin3d.com; see Appendix 2, available at www.cmaj.ca/lookup/suppl/doi:10.1503 /cmaj.131042/-/DC1, for interpretation of this patient's results).

A history of BCG vaccination in infancy can be disregarded in people 10 years or older, because it is associated with a low rate of positive results on the tuberculin skin test in adults. ${ }^{2}$ A history of BCG vaccination should also be ignored in patients with a high probability of TB infection or a high risk of disease progression. ${ }^{2}$ When $\mathrm{BCG}$ assist with interpretation of tuberculin skin test
Competing interests: None declared.

This article has been peer reviewed.

Correspondence to: Paul Bunce, paul.bunce@uhn.ca

CMAJ 2014. DOI:10.1503 /cmaj.131042 
status is unknown but may be relevant in interpretation of the tuberculin skin test, The BCG World Atlas (www.bcgatlas.org) can be consulted for information on current and past BCG policies in a variety of countries. ${ }^{5}$

\section{Given a positive tuberculin skin test, what are the initial steps in managing this patient's treatment?}

First and foremost, active TB infection must be excluded through history, physical examination and chest radiography. If there is clinical or radiologic suspicion of pulmonary TB, sputum samples should be sent for acid-fast bacilli smear and culture. ${ }^{3}$

Once active TB is ruled out, the patient should be given treatment for latent TB infection. Standard therapy involves a nine-month course of isoniazid (INH; $5 \mathrm{mg} / \mathrm{kg}$ per day to a maximum of $300 \mathrm{mg}$ per day), although alternative regimens exist. ${ }^{3}$ Daily pyridoxine (vitamin $\mathrm{B}_{6}$ ) is recommended for patients at risk of neuropathy (e.g., those with diabetes or renal insufficiency), but is generally offered to all patients to prevent INH-related peripheral neuropathy. ${ }^{3}$ In most Canadian health jurisdictions, medications for the treatment of TB are provided to patients free of charge. Suggestions for when to refer are listed in Box 1.

\section{What are the risks of treatment and how should this patient be monitored while undergoing therapy?}

The patient should be advised to avoid alcohol, and the INH-related risks of hepatotoxicity, neuropathy, rash, gastrointestinal effects and drug interactions should be explained. Hepatitis is rare among patients under 20 years of age, but the risk increases to about $2 \%$ or more in those over 50 years of age. ${ }^{3.6}$ Previous liver disease, regular alcohol consumption and concomitant use of other

Box 1: Consider referral to a clinician with experience managing tuberculosis in the following situations*

- New TB exposure in a patient with a prior positive tuberculin skin test

- Positive tuberculin skin test and

- Prior BCG vaccination in childhood (after age $1 \mathrm{yr}$ ) or adulthood

- Contact with drug-resistant TB

- Acute or active liver disease

- Allergy or intolerance to INH

- History of INH-induced hepatitis

Pregnancy

- Current INH-related hepatotoxicity (as defined above)

- Uncertainty of the practitioner regarding any aspect of diagnosis and management of latent TB infection

- Management of active TB

Note: $\mathrm{BCG}$ = bacille Calmette-Guérin, INH = isoniazid, TB = tuberculosis.

* Recommendations based on expert opinion and Canadian TB guidelines. ${ }^{3}$ hepatotoxic drugs also increase the risk of hepatitis. Although INH is considered safe in pregnancy and breastfeeding, there is an increased risk of hepatotoxicity in the first three months postpartum; current guidelines recommend deferral of INH treatment until three months after delivery. ${ }^{3}$

Baseline measurement of liver enzymes (i.e., aspartate transaminase [AST] and alanine transaminase [ALT]) and bilirubin, and monthly clinical monitoring for hepatotoxicity (e.g., nausea, vomiting, abdominal pain, dark-coloured urine and jaundice) are recommended. ${ }^{3}$ Serum transaminase and bilirubin levels should be measured in patients who become symptomatic, and should be monitored monthly in patients who are over 50 years of age, have pre-existing liver disease or risk factors for liver disease, or are pregnant. ${ }^{3}$ Isoniazid should be held, at least temporarily, if AST or ALT levels exceed five times the upper limit of normal in the absence of symptoms, or three times the upper limit with clinical symptoms. ${ }^{3,6}$ Evaluation for other causes of acute hepatitis should also be investigated. Referral to a TB specialist, restarting INH after liver enzymes have returned to normal (if enzymes were not severely elevated over 1000 $\mathrm{U} / \mathrm{L}$ ), or starting a second-line regimen are all reasonable options for subsequent management. ${ }^{3}$ There is no recommended routine follow-up after completion of treatment for latent TB infection. ${ }^{3}$

\section{Case revisited}

The patient started INH therapy and, shortly thereafter, hemodialysis was initiated; latent TB treatment should not delay life-saving treatments, such as hemodialysis. Routine monitoring at two months showed a mild increase in her AST and ALT levels, but she remained asymptomatic. She completed a nine-month INH course without further complication.

\section{References}

1. Canadian Thoracic Society of the Canadian Lung Association and Public Health Agency of Canada. Chapter 2, Canadian tuberculosis standards, 7th ed. Can Respir J 2013;20(Suppl A):9A-15A.

2. Canadian Thoracic Society of the Canadian Lung Association and Public Health Agency of Canada. Chapter 4, Canadian tuberculosis standards, 7th ed. Can Respir J 2013;20(Suppl A):23A-34A.

3. Canadian Thoracic Society of the Canadian Lung Association and Public Health Agency of Canada. Chapter 6, Canadian tuberculosis standards, 7th ed. Can Respir J 2013;20(Suppl A):44A-53A.

4. Horsburgh CR, Rubin EJ. Latent tuberculosis infection in the United States. N Engl J Med 2011;364:1441-8.

5. The BCG world atlas [online database]. Montréal (QC): McGill University. Available: www.bcgatlas.org/ (accessed 2013 Oct. 16).

6. Saukkonen JJ, Cohn DL, Jasmer RM, et al. An official ATS statement: hepatotoxicity of antituberculous therapy. Am J Respir Crit Care Med 2006;174:935-52.

Affiliations: Department of Family and Community Medicine (Redditt), University of Toronto; Department of Medicine (Bunce), University of Toronto; Division of Infectious Diseases (Bunce), University Health Network, Toronto, Ont.

Contributors: Both authors wrote and revised the article, and approved the version submitted for publication. 